Document downloaded from:

http://hdl.handle.net/10251/121062

This paper must be cited as:

Singh, S.; Gupta, D.; Singh, R.; Singh, M.; Martínez Molada, E. (2018). Convergence of an Iteration of Fifth-Order Using Weaker Conditions on First Order Fréchet Derivative in Banach Spaces. International Journal of Computational Methods. 15(6):1-18. https://doi.org/10.1142/S0219876218500482

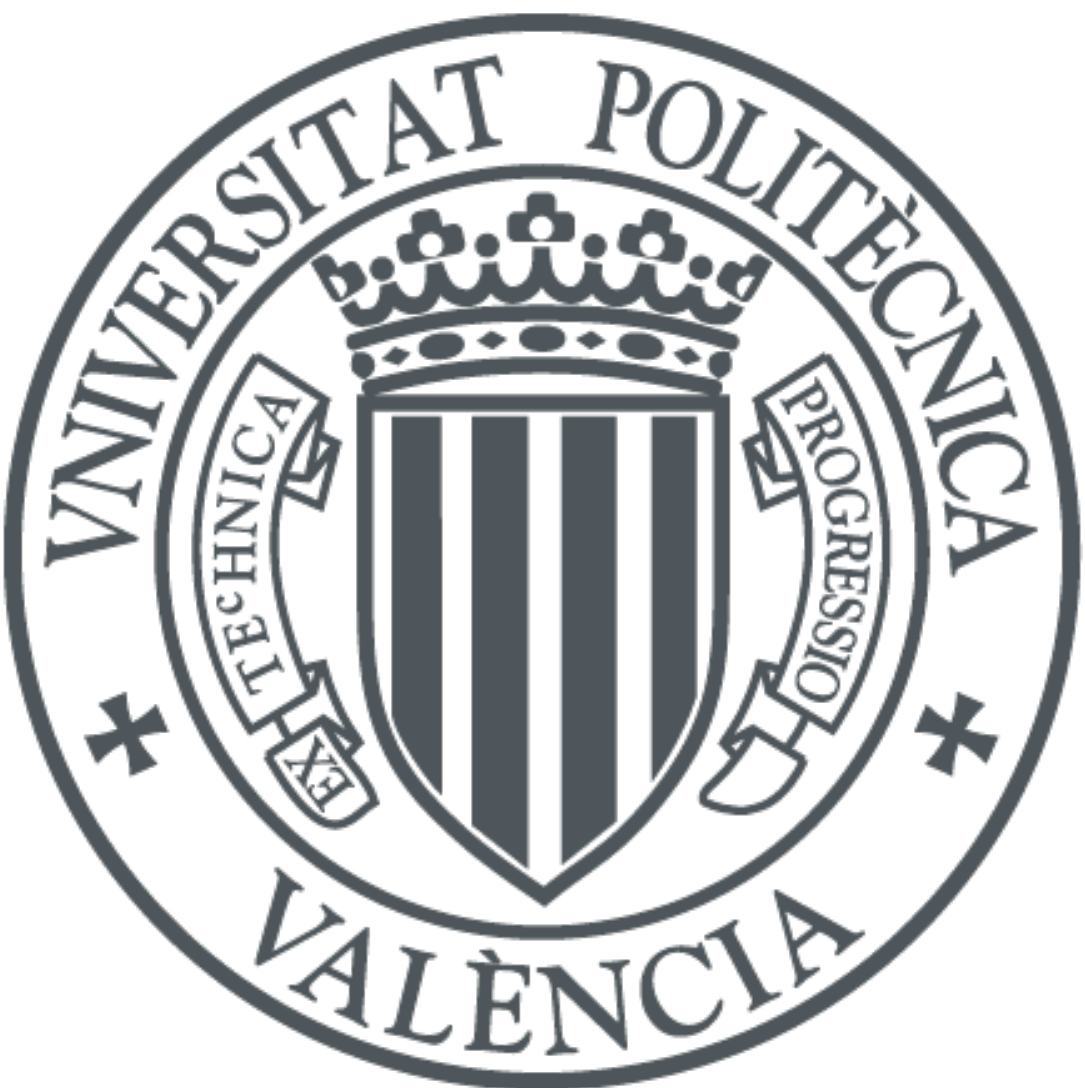

The final publication is available at

http://doi.org/10.1142/S0219876218500482

Copyright World Scientific

Additional Information 


\title{
Convergence of an iteration of fifth-order using weaker conditions on first order Fréchet derivative in Banach spaces
}

\author{
S. Singh ${ }^{1}$, D.K. Gupta ${ }^{2}$ R. Singh ${ }^{3}$, M. Singh ${ }^{4}$ and E. Martinez ${ }^{5}$ \\ Department of Mathematical Sciences, I.K.G. Punjab Technical University Jalandhar \\ Department of Mathematics, I.I.T. Kharagpur \\ Department of Mathematics, Birla Institute of technology, Mesra \\ Department of Mathematical Modelling, Statistics and Bioinformatics, Ghent University \\ Instituto de Matemática Pura y Aplicada, Universitat Politècnica de València
}

\begin{abstract}
The convergence analysis both local under weaker Argyros-type conditions and semilocal under $\omega$-condition is established using first order Fréchet derivative for an iteration of fifth order in Banach spaces. This avoids derivatives of higher orders which are either difficult to compute or do not exist at times. The Lipchitz and the Hölder conditions are particular cases of the $\omega$-condition. Examples can be constructed for which the Lipchitz and Hölder conditions fail but the $\omega$-condition holds. Recurrence relations are used for the semilocal convergence analysis. Existence and uniqueness theorems and the error bounds for the solution are provided. Different examples are solved and convergence balls for each of them are obtained. These examples include Hammerstein-type integrals to demonstrate the applicability of our approach.
\end{abstract}

Keywords: Local convergence; Semilocal convergence; Hammerstein-type integral equation; Argyros-type conditions; Fréchet derivative.

Mathematical Subject Classification 65H10, 47H99

\section{Introduction}

Many challenging problems in science and engineering require obtaining solutions of nonlinear equations. It is extensively studied as mathematical modeling of many real-world problems $[9,10,27]$ involves differential equations, difference equations, integral equations, etc. which leads to solving these equations. Consider

$$
F(x)=0,
$$

\footnotetext{
${ }^{1}$ Corresponding* sukhjitmath@gmail.com

${ }^{2} \mathrm{dkg} @$ maths.iitkgp.ernet.in

${ }^{3}$ randhir.math@gmail.com

${ }^{4}$ singhmehakpreet@gmail.com

${ }^{5}$ eumarti@mat.upv.es
} 
where, $F: D \subseteq X \rightarrow Y$ be twice differentiable nonlinear operator in an open convex region $D_{0} \subseteq D$ for Banach spaces $X$ and $Y$. Generally, one point and multi points iterative methods along with their convergence analysis and estimation of error bounds are used for solving (1.1). Local convergence analysis [21, 23, 28, 29, 33] involves the information about the solution whereas the semilocal convergence analysis $[7,15,5,6,22,25,26,30,32]$ uses information about the initial point. The quadratically convergent Newton's method used for solving (1.1) is given for $n=0,1,2, \ldots$ by

$$
x_{n+1}=x_{n}-\Gamma_{n} F\left(x_{n}\right),
$$

where, $\Gamma_{n}=F^{\prime}\left(x_{n}\right)^{-1}$ and $x_{0}$ is the starting point. Kantorovich [27] established sufficient conditions for its convergence by using either majorizing sequences or recurrence relations. But recurrence relations are often used as problems in Banach spaces here are changed to simpler problems with real sequences and functions. Higher order iterative methods and their convergence analysis are also used for solving (1.1). It is worth mentioning that higher order methods require higher computational cost. Also, they involve derivatives of higher order which are either expensive to evaluate or become unbounded. Thus, they are only of theoretical interest.

Recently, higher order iterative methods without using higher order derivatives for solving (1.1) have also gained importance with the advancement of technology and development of fast computers. Thus, they are useful for applications which require quick convergence. The local convergence of a method of third order using Argyros-type conditions on $F^{\prime}$ is discussed in [17]. For a third order family of iterations, it is described in [18] for the Lipschitz condition on $F^{\prime}$. It is given for $n \geq 0$, by

$$
\begin{aligned}
y_{n} & =x_{n}-\theta \Gamma_{n} F\left(x_{n}\right), \\
x_{n+1} & =x_{n}-\frac{\theta^{2}+\theta-1}{\theta^{2}} \Gamma_{n} F\left(x_{n}\right)-\frac{1}{\theta^{2}} \Gamma_{n} F\left(y_{n}\right),
\end{aligned}
$$

where, $\theta \in \mathbb{R}-\{0\}$ and $x_{0}$ is the starting point. Its semilocal convergence analysis using same condition is given in [19]. For $\theta=1$, its semilocal convergence is done in [31] under Hölder condition on $F^{\prime}$. For Halley-type iterations, it is performed under the Lipschitz condition on $F^{\prime}$ in $[13,14]$. For the variants of the Chebyshev-Halley iteration family, it is studied in [16] under the Hölder condition on $F^{\prime}$. The local convergence analysis of deformed Halley method on $F^{\prime}$ under Hölder condition is developed in [24]. Likewise, a number of iterations of fourth order with convergence analysis using recurrence relations are also developed for solving (1.1) under various conditions. Hueso and Martínez [12] performed the semilocal convergence of a family of iterations of higher order under the Lipschitz condition on $F^{\prime \prime}$. It is given for $n \geq 0$, 
by

$$
\begin{aligned}
y_{n} & =x_{n}-\theta \Gamma_{n} F\left(x_{n}\right), \\
z_{n} & =y_{n}-\Gamma_{n} F\left(y_{n}\right), \\
x_{n+1} & =z_{n}-\Gamma_{n} F\left(z_{n}\right),
\end{aligned}
$$

where, $\theta \in \mathbb{R}-\{0\}$ and $x_{0}$ is the starting point. For $\theta= \pm 1$, it leads to fourth order iterative methods. Its local convergence analysis is carried out assuming the Lipschitz condition on $F^{\prime}$ in $[20]$.

Recently, Hernández and Martínez [4] developed the semilocal convergence of (1.4) for $\theta=1$ under the $\omega$-condition on $F^{\prime}$. This condition is given for a continuous and non-decreasing function $\omega: R_{+} \rightarrow R_{+}$such that $\omega(0) \geq 0$ by $\left\|F^{\prime}(x)-F^{\prime}(y)\right\| \leq \omega(\|x-y\|), \forall x, y \in D$. This condition generalizes the Lipschitz/Hölder condition given by $\omega(x)=N x$ and $\omega(x)=N x^{\alpha}, \alpha \in$ $(0,1]$.

We shall describe the convergence analysis, both local and semilocal, for an iteration of fifth order discussed in [8] using weaker Argyros-type and $\omega$-conditions on first order Fréchet derivative for solving (1.1) in Banach spaces. It is given for $n=0,1,2, \ldots$, by

$$
\begin{aligned}
y_{n} & =x_{n}-\Gamma_{n} F\left(x_{n}\right), \\
z_{n} & =y_{n}-5 \Gamma_{n} F\left(y_{n}\right), \\
x_{n+1} & =z_{n}-\frac{1}{5} \Gamma_{n}\left[-16 F\left(y_{n}\right)+F\left(z_{n}\right)\right],
\end{aligned}
$$

where, $x_{0}$ is the starting point. Using recurrence relations, its convergence under Lipschitz and Hölder conditions on $F^{\prime}$ are provided in $[1,2,3]$. Clearly, the Lipschitz and Hölder conditions on $F^{\prime}$ are not satisfied for the following nonlinear Hammerstein type [11] integral equation

$$
x(r)+\sum_{i=1}^{m} \int_{a}^{b} K_{i}(r, s) S_{i}(x(s)) d s=f(r), r \in[a, b],
$$

where functions $f, K_{i}$ and $S_{i}$ for $i=1,2, \ldots m$ are known, the solution $x$ is to be determined and $-\infty<a<b<+\infty$. In order to solve (1.6), we have to solve

$$
F(x)(r)=x(r)+\sum_{i=1}^{m} \int_{a}^{b} K_{i}(r, s) S_{i}(x(s)) d s-f(r) .
$$

If $S_{i}^{\prime}(x(s))$ is $\left(M_{i}, \alpha_{i}\right)$ - Hölder continuous in $D$, then, under max-norm, we have

$$
\left\|F^{\prime}(x)-F^{\prime}(y)\right\| \leq \sum_{i=1}^{m} M_{i}\|x-y\|^{\alpha_{i}}, M_{i} \geq 0, \alpha_{i} \in[0,1], \forall x, y \in D .
$$

For different $\alpha_{i}, F^{\prime}$ neither satisfies Lipschitz nor Hölder condition but satisfies the weaker $\omega$-condition. 
The rest of the paper is arranged as follows. In section 2, the local convergence under weaker Argyros-type condition on $F^{\prime}$ for an iteration of fifth-order in Banach spaces is described. The convergence is performed by deriving recurrence relations. The existence and uniqueness theorems and the error bounds for the solution are provided. Different numerical examples are also solved here. Section 3 is devoted to the semilocal convergence analysis under weaker $\omega$-condition on $F^{\prime}$ for the same iteration. Here also, the recurrence relations are used. The existence and uniqueness theorems and error bounds for the solution are provided. Different numerical examples are solved. Finally, conclusions are included in section 4.

\section{Local convergence analysis}

Here, we shall discuss the local convergence of (1.5) for solving (1.1) under weaker Argyros-type condition on $F^{\prime}$ in Banach spaces. Let $x^{*} \in D, F\left(x^{*}\right)=0, F^{\prime}\left(x^{*}\right)^{-1}$ exists and $F^{\prime}$ satisfies the following Argyros-type conditions for strictly increasing functions $f_{0}$ and $g_{0}$ defined on the interval $[0, \infty)$ with values in $[0, \infty)$

$$
\begin{aligned}
\left\|F^{\prime}\left(x^{*}\right)^{-1}\left(F^{\prime}(x)-F^{\prime}(y)\right)\right\| & \leq f_{0}(\|x-y\|), x, y \in D . \\
\left\|F^{\prime}\left(x^{*}\right)^{-1}\left(F^{\prime}(x)-F^{\prime}\left(x^{*}\right)\right)\right\| & \leq g_{0}\left(\left\|x-x^{*}\right\|\right), x \in D .
\end{aligned}
$$

where $f_{0}(0)=0$, and $g_{0}(0)=0$. Define a parameter $\rho_{0}$ by

$$
\rho_{0}=\left\{u \geq 0 ; g_{0}(u)<1\right\}
$$

For local convergence analysis, following theorem is given.

Theorem 2.1. Let $x^{*} \in D$ and $F^{\prime}\left(x^{*}\right)^{-1}$ exists such that (2.1) and (2.2) hold. If $g_{0}(\rho)<1$ then the sequence $\left\{x_{n}\right\}$ obtained by (1.5) is defined, conatined in $B\left(x^{*}, \rho\right)$ and converges to $x^{*}$ for $x_{0} \in B\left(x^{*}, \rho\right)=\left\{x \in X:\left\|x-x^{*}\right\|<\rho\right\} \subset D$ for some suitable radius $\rho$. Also, the error bounds for all $n \geq 0$ are

$$
\begin{aligned}
\left\|y_{n}-x^{*}\right\| & \leq h_{1}\left(\left\|x_{n}-x^{*}\right\|\right)\left\|x_{n}-x^{*}\right\|<\left\|x_{n}-x^{*}\right\|<\rho, \\
\left\|z_{n}-x^{*}\right\| & \leq h_{2}\left(\left\|x_{n}-x^{*}\right\|\right)\left\|x_{n}-x^{*}\right\|<\rho, \\
\left\|x_{n+1}-x^{*}\right\| & \leq h_{3}\left(\left\|x_{n}-x^{*}\right\|\right)\left\|x_{n}-x^{*}\right\|<\rho,
\end{aligned}
$$

where, $h_{1}, h_{2}$ and $h_{3}$ are the functions to be defined.

Proof. Using $x_{0} \in D$ and from (2.2), we get

$$
\left\|I-F^{\prime}\left(x^{*}\right)^{-1} F^{\prime}\left(x_{0}\right)\right\| \leq g_{0}\left(\left\|x_{0}-x^{*}\right\|\right) \leq g_{0}(\rho)<1 .
$$

Thus, $\Gamma_{0}$ exists and using Banach Lemma for invertible operators, we get

$$
\left\|\Gamma_{0} F^{\prime}\left(x^{*}\right)\right\| \leq \frac{1}{1-g_{0}\left(\left\|x_{0}-x^{*}\right\|\right)} .
$$


Therefore, $y_{0}$ is well defined and hence $z_{0}$ and $x_{1}$ are well defined. For $n=0$ and using (1.5), we get

$$
y_{0}-x^{*}=-\Gamma_{0} F^{\prime}\left(x^{*}\right) \int_{0}^{1} F^{\prime}\left(x^{*}\right)^{-1}\left[F^{\prime}\left(x^{*}+\theta\left(x_{0}-x^{*}\right)\right)-F^{\prime}\left(x_{0}\right)\right]\left(x_{0}-x^{*}\right) d \theta .
$$

Taking norm on both sides and using (2.1) and (2.6), we get

$$
\begin{aligned}
\left\|y_{0}-x^{*}\right\| & \leq \frac{\int_{0}^{1} f_{0}\left(|1-\theta|\left\|x_{0}-x^{*}\right\|\right) d \theta}{\left(1-g_{0}\left(\left\|x_{0}-x^{*}\right\|\right)\right)}\left\|x_{0}-x^{*}\right\| \\
& \leq h_{1}\left(\left\|x_{0}-x^{*}\right\|\right)\left\|x_{0}-x^{*}\right\|,
\end{aligned}
$$

where,

$$
h_{1}(u)=\frac{\int_{0}^{1} f_{0}(|1-\theta| u) d \theta}{\left(1-g_{0}(u)\right)} .
$$

Let $g_{1}(u)=h_{1}(u)-1$. Then $g_{1}(0)=-1$ and $g_{1}\left(\rho_{0}^{-}\right) \rightarrow \infty$. Therefore, $g_{1}(u)$ has a minimum positive root $r_{1}$ in $\left[0, \rho_{0}\right)$. Thus, for $u \in\left[0, r_{1}\right)$, we get $0 \leq h_{1}(u)<1$ and

$$
\left\|y_{0}-x^{*}\right\| \leq h_{1}\left(\left\|x_{0}-x^{*}\right\|\right)\left\|x_{0}-x^{*}\right\|<\left\|x_{0}-x^{*}\right\| .
$$

Again, for $n=0$ and using (1.5), we get

$$
\left\|z_{0}-x^{*}\right\| \leq\left\|y_{0}-x^{*}\right\|+5\left\|F^{\prime}\left(x^{*}\right)^{-1} F\left(y_{0}\right)\right\|\left\|\Gamma_{0} F^{\prime}\left(x^{*}\right)\right\| .
$$

Since,

$$
\begin{aligned}
\left\|F^{\prime}\left(x^{*}\right)^{-1} F\left(y_{0}\right)\right\| & \leq\left\|\int_{0}^{1} F^{\prime}\left(x^{*}\right)^{-1}\left(F^{\prime}\left(x^{*}+\theta\left(y_{0}-x^{*}\right)\right)-F^{\prime}\left(x^{*}\right)\right) d \theta+I\right\|\left\|\left(y_{0}-x^{*}\right)\right\| \\
& \leq\left(1+\int_{0}^{1} g_{0}\left(|\theta|\left\|y_{0}-x^{*}\right\|\right) d \theta\right)\left\|\left(y_{0}-x^{*}\right)\right\|
\end{aligned}
$$

and on using (2.6) and (2.10), we get

$$
\begin{aligned}
\left\|z_{0}-x^{*}\right\| & \leq\left(1+5\left(\frac{1+\int_{0}^{1} g_{0}\left(|\theta|\left\|y_{0}-x^{*}\right\|\right) d \theta}{1-g_{0}\left(\left\|\left(x_{0}-x^{*}\right)\right\|\right)}\right)\right)\left\|y_{0}-x^{*}\right\| \\
& \leq\left(1+\frac{5\left(1+\int_{0}^{1} g_{0}\left(|\theta| h_{1}\left(\left\|x_{0}-x^{*}\right\|\right)\left\|x_{0}-x^{*}\right\|\right) d \theta\right)}{1-g_{0}\left(\left\|x_{0}-x^{*}\right\|\right)}\right) h_{1}\left(\left\|x_{0}-x^{*}\right\|\right)\left\|x_{0}-x^{*}\right\| \\
& \leq h_{2}\left(\left\|x_{0}-x^{*}\right\|\right)\left\|x_{0}-x^{*}\right\| .
\end{aligned}
$$

where,

$$
h_{2}(u)=\left(1+\frac{5\left(1+\int_{0}^{1} g_{0}\left(|\theta| h_{1}(u) u\right) d \theta\right)}{1-g_{0}(u)}\right) h_{1}(u)
$$


Let $g_{2}(u)=h_{2}(u)-1$. Then $g_{2}(0)=-1$ and $g_{2}\left(\rho_{0}^{-}\right) \rightarrow \infty$. Therefore, $g_{2}(u)$ has a minimum positive root $r_{2}$ in $\left[0, \rho_{0}\right)$. Then for $u \in\left[0, r_{2}\right)$, we get $0 \leq h_{2}(u)<1$ and

$$
\left\|z_{0}-x^{*}\right\| \leq h_{2}\left(\left\|x_{0}-x^{*}\right\|\right)\left\|x_{0}-x^{*}\right\|<\left\|x_{0}-x^{*}\right\| .
$$

Again, from (1.5) for $n=0$, and by using (2.6) and (2.10), we get

$$
\begin{aligned}
\left\|x_{1}-x^{*}\right\| \leq & \left\|z_{0}-x^{*}\right\|+\frac{1}{5}\left\|\Gamma_{0} F^{\prime}\left(x^{*}\right)\right\|\left(16\left\|F^{\prime}\left(x^{*}\right)^{-1} F\left(y_{0}\right)\right\|+\left\|F^{\prime}\left(x^{*}\right)^{-1} F\left(z_{0}\right)\right\|\right) \\
\leq & \left(h_{2}\left(\left\|x_{0}-x^{*}\right\|\right)+\frac{1}{5} \frac{1}{1-g_{0}\left(\left\|x_{0}-x^{*}\right\|\right)}\left(16\left(1+\int_{0}^{1} g_{0}\left(|\theta| h_{1}\left(\left\|x_{0}-x^{*}\right\|\right)\left\|x_{0}-x^{*}\right\|\right) d \theta\right)\right.\right. \\
& \left.\left.h_{1}\left(\left\|x_{0}-x^{*}\right\|\right)+\left(1+\int_{0}^{1} g_{0}\left(|\theta| h_{2}\left(\left\|x_{0}-x^{*}\right\|\right)\left\|x_{0}-x^{*}\right\|\right) d \theta\right) h_{2}\left(\left\|x_{0}-x^{*}\right\|\right)\right)\right)\left\|x_{0}-x^{*}\right\| \\
\leq & h_{3}\left(\left\|x_{0}-x^{*}\right\|\right)\left\|x_{0}-x^{*}\right\|
\end{aligned}
$$

where,

$h_{3}(u)=\left(h_{2}(u)+\frac{1}{5} \frac{1}{1-g_{0}(u)}\left(16 h_{1}(u)\left(1+\int_{0}^{1} g_{0}\left(|\theta| h_{1}(u) u\right) d \theta\right)+\left(1+\int_{0}^{1} g_{0}\left(|\theta| h_{2}(u) u\right) d \theta\right) h_{2}(u)\right)\right)$.

Let $g_{3}(u)=h_{3}(u)-1$. Then $g_{3}(0)=-1$ and $g_{3}\left(\rho_{0}^{-}\right) \rightarrow \infty$. Therefore, $g_{3}(u)$ has a minimum positive root $r_{3}$ in $\left[0, \rho_{0}\right)$. Then for $u \in\left[0, r_{3}\right)$, we get $0 \leq h_{3}(u)<1$ and

$$
\left\|x_{1}-x^{*}\right\| \leq h_{3}\left(\left\|x_{0}-x^{*}\right\|\right)\left\|x_{0}-x^{*}\right\|<\left\|x_{0}-x^{*}\right\| .
$$

Let $\rho=\min \left\{r_{1}, r_{2}, r_{3}\right\}$. Then, for $u \in[0, \rho)$, we get $0 \leq h_{1}(u)<1,0 \leq h_{2}(u)<1$ and $0 \leq$ $h_{3}(u)<1$. Thus, theorem is proved for $n=0$. By replacing $x_{0}, y_{0}, z_{0}$ and $x_{1}$ by $x_{n}, y_{n}, z_{n}, x_{n+1}$ in the preceding way, we get the error bounds given by (2.3)-(2.5). Using the estimate $\| x_{n+1}-$ $x^{*}\|<\| x_{n}-x^{*} \|<\rho$, we get $x_{n+1} \in B\left(x^{*}, \rho\right)$. Clearly, $h_{3}(u)$ is an increasing function in its domain and since, $h_{3}(u)<1 \forall u \in[0, \rho)$, this gives

$$
\begin{aligned}
\left\|x_{n+1}-x^{*}\right\| & \leq h_{3}(u)\left\|x_{n}-x^{*}\right\| \leq h_{3}(u) h_{3}\left(\left\|x_{n-1}-x^{*}\right\|\right)\left\|x_{n-1}-x^{*}\right\| \\
& \leq h_{3}(u)^{2} h_{3}\left(\left\|x_{n-2}-x^{*}\right\|\right)\left\|x_{n-2}-x^{*}\right\| \leq \ldots \leq h_{3}(u)^{n+1}\left\|x_{0}-x^{*}\right\| .
\end{aligned}
$$

Thus, $\lim _{n \rightarrow \infty} x_{n}=x^{*}$ as $\lim _{n \rightarrow \infty} h_{3}(u)^{n+1}=0$.

\subsection{Numerical examples}

In this subsection, we have worked out a number of examples to demonstrate the applicability of our approach.

Example 2.1. Let $X=Y=\mathbb{R}$ and define $F$ on $D=(-1,1)$ by $F(x)=\sin x+x^{7 / 4}$.

It is obvious that $x^{*}=0$. For $x, y \in D$, we get $\left\|F^{\prime}\left(x^{*}\right)^{-1}\left(F^{\prime}(x)-F^{\prime}(y)\right)\right\| \leq\left(\|x-y\|+\frac{7}{4}\|x-y\|^{3 / 4}\right)$.

It is easy to see that neither Lipschitz nor Hölder conditions hold on $F^{\prime}$. Clearly, $f_{0}(x)=$ $x+\frac{7}{4} x^{3 / 4}$ and $g_{0}(x)=x+\frac{7}{4} x^{3 / 4}$. Thus, weaker Argyros-type conditions hold on $F^{\prime}$. By applying Theorem 2.1, $x^{*} \in B(0,0.023251)$. 
Example 2.2. Let $X=Y=\mathbb{R}$ and define $F$ on $D=(-1,1)$ by $F(x)=e^{x}-1$.

Clearly, $x^{*}=0$. For $x, y \in D$, we have $\left\|F^{\prime}\left(x^{*}\right)^{-1}\left(F^{\prime}(x)-F^{\prime}(y)\right)\right\| \leq e\|x-y\|$. Thus, $F^{\prime}$ satisfies both Lipschitz and Hölder conditions. Clearly, $f_{0}(x)=e x$ and $g_{0}(x)=(e-1) x$. Thus, weaker Argyros-type conditions also hold. By applying Theorem 2.1, $x^{*} \in B(0,0.05708)$.

Example 2.3. Consider the nonlinear Hammerstein type integral equation given by

$$
F(x)(r)=x(r)-5 \int_{0}^{1} r s x(s)^{1+\alpha} d s, \alpha \in(0,1)
$$

with $x(r)$ in $\mathcal{C}[0,1]$.

Clearly, $x^{*}=0$. For $x, y \in D$, we have $\left\|F^{\prime}\left(x^{*}\right)^{-1}\left(F^{\prime}(x)-F^{\prime}(y)\right)\right\| \leq 2.5(1+\alpha)\|x-y\|^{\alpha}$. Thus, Hölder condition holds on $F^{\prime}$. Clearly, $f_{0}(x)=2.5(1+\alpha) x^{\alpha}$ and $g_{0}(x)=2.5(1+\alpha) x^{\alpha}$. Thus, weaker Argyros-type conditions also hold. Taking $\alpha=1 / 2$ and by applying Theorem $2.1, x^{*} \in B(0,0.00087)$.

\section{Semilocal convergence}

Here, we shall discuss the semilocal convergence analysis using recurrence relations of (1.5) for solving (1.1) under weaker $\omega$-condition on $F^{\prime}$ in Banach spaces. First of all, some preliminary results are given. Next, recurrence relations are derived. The existence, uniqueness theorems and error bounds for the solution are also provided. Finally, different examples are solved to demonstrate the efficacy of our approach.

\subsection{Preliminary results}

In this subsection, we shall describe some preliminary results used for the convergence of (1.5) for solving (1.1). Let $x_{0} \in D$ and $\Gamma_{0}=F^{\prime}\left(x_{0}\right)^{-1} \in \mathcal{L}(Y, X)$ exists, where $\mathcal{L}(Y, X)$ denotes the set of bounded linear operators from $Y$ to $X$. Let the following conditions hold.

(1) $\left\|\Gamma_{0}\right\| \leq \beta$,

(2) $\left\|\Gamma_{0} F\left(x_{0}\right)\right\| \leq \eta$,

(3) $\quad\left\|F^{\prime}(x)-F^{\prime}(y)\right\| \leq \omega(\|x-y\|), x, y \in D$, for a continuous non-decreasing real function $\omega(x), x>0, \omega(0) \geq 0$ such that, $\omega(t x) \leq t^{\alpha} \omega(x)$ for $t \in[0,1], x \in(0, \infty), \alpha \in[0,1]$.

Let $b_{0}=\beta w(\eta)$, and define the sequence $\left\{b_{n}\right\}$, where,

$$
\begin{gathered}
b_{n+1}=b_{n} \phi\left(b_{n}\right)^{1+\alpha} \psi\left(b_{n}\right)^{\alpha}, \quad n \geq 0, \\
\phi(u)=\frac{1}{1-u(1+\psi(u))^{\alpha}}, \\
\psi(u)=\left(\frac{u}{1+\alpha}+(u+1) \xi(u)+\left(\frac{u}{1+\alpha}\right) \xi(u)^{1+\alpha}\right),
\end{gathered}
$$


and

$$
\xi(u)=\left(\frac{4 u}{5(1+\alpha)}+\frac{u}{5(1+\alpha)}\left(1+\frac{5 u}{1+\alpha}\right)^{1+\alpha}\right) .
$$

In order to investigate the convergence of $\left\{x_{n}\right\}$ generated by (1.5) to a solution of (1.1), we have to show that $\left\{x_{n}\right\}$ is a Cauchy sequence. For this, we have to study the properties of $\left\{b_{n}\right\}$. The following lemmas are proved to study the properties of the sequence $\left\{b_{n}\right\}$.

Lemma 3.1. Let $\phi(u), \psi(u)$ and $\xi(u)$ be the functions given by (3.2)-(3.4) and $s_{0}$ is the minimum positive root of $h(u)=u(1+\xi(u))^{\alpha}-1$ in $(0,1)$. Then

(i) $\phi(u)$ is increasing and $\phi(u)>1$ for $u \in\left(0, s_{0}\right)$,

(ii) $\psi(u)$ and $\xi(u)$ are the increasing functions for $u \in\left(0, s_{0}\right)$.

Proof. The proof is trivial.

Consider the auxiliary function

$$
r(u)=\left(\frac{u}{1+\alpha}+(u+1) \xi(u)+\frac{u \xi(u)^{1+\alpha}}{1+\alpha}\right)^{\alpha}-\left(1-u(1+\xi(u))^{\alpha}\right)^{1+\alpha} .
$$

Clearly, $r(0)<0, r\left(s_{0}\right)>0$ and $r^{\prime}(u)>0$. Thus, $r(u)$ has a real root $s_{1}$ in $\left(0, s_{0}\right)$.

Lemma 3.2. Let $\phi(u), \psi(u)$ and $\xi(u)$ be the functions defined by (3.2)-(3.4), respectively. If $b_{0} \in\left(0, s_{1}\right)$, then

(i) $\phi\left(b_{0}\right)^{1+\alpha} \psi\left(b_{0}\right)^{\alpha}<1$,

(ii) $\phi\left(b_{0}\right) \psi\left(b_{0}\right)<1$,

(iii) The sequence $\left\{b_{n}\right\}$ is decreasing and $b_{n}<s_{1}$ for all $n \geq 0$,

(iv) $b_{n}\left(1+\xi\left(b_{n}\right)\right)^{p}<1$.

Proof. Taking $u=b_{0}$ in $r(u)$, we get $\phi\left(b_{0}\right)^{1+\alpha} \psi\left(b_{0}\right)^{\alpha}<1$ for all $b_{0} \in\left(0, s_{1}\right)$. Since $\phi\left(b_{0}\right)>1$, this gives $\left(\phi\left(b_{0}\right) \psi\left(b_{0}\right)\right)^{\alpha}<1$, and hence $\phi\left(b_{0}\right) \psi\left(b_{0}\right)<1$. To prove (iii), we use mathematical induction on (3.1). For $n=0$, we get $b_{1}=b_{0} \phi\left(b_{0}\right)^{1+\alpha} \psi\left(b_{0}\right)^{\alpha}<b_{0}$. Assume that $b_{k}<b_{k-1}$ for $k \leq n$. Since $\phi(u)$ and $\psi(u)$ are increasing functions, we get $b_{n+1}=b_{n} \phi\left(b_{n}\right)^{1+\alpha} \psi\left(b_{n}\right)^{\alpha}<$ $b_{n-1} \phi\left(b_{n-1}\right)^{1+\alpha} \psi\left(b_{n-1}\right)^{\alpha}=b_{n}$. Hence, $\left\{b_{n}\right\}$ is a decreasing and $b_{n}<s_{1}$ for all $n \geq 0$. Since $\xi(u)$ is an increasing function, we get $b_{n}\left(1+\xi\left(b_{n}\right)\right)^{\alpha}<b_{n-1}\left(1+\xi\left(b_{n-1}\right)\right)^{\alpha}<b_{0}\left(1+\xi\left(b_{0}\right)\right)^{\alpha}<$ $1 \forall b_{0} \in\left(0, s_{1}\right)$. This proves (iv) as $b_{n}\left(1+\xi\left(b_{n}\right)\right)^{\alpha}<1$ for all $n \geq 0$ and $b_{0} \in\left(0, s_{1}\right)$.

\subsection{Recurrence relations}

In this subsection, we shall establish the recurrence relations for (1.5) under the assumptions $(1),(2)$ and (3) described in previous subsection. The existence of $\Gamma_{0}$ gives the existence of $y_{0}$ and hence $\left\|\Gamma_{0}\right\| \omega\left(\left\|y_{0}-x_{0}\right\|\right) \leq \beta \omega(\eta)=b_{0}$. Taking $n=0$, in (1.5), we get

$$
z_{0}-x_{0}=y_{0}-x_{0}-5 \Gamma_{0} \int_{0}^{1}\left(F^{\prime}\left(x_{0}+t\left(y_{0}-x_{0}\right)\right)-F^{\prime}\left(x_{0}\right)\right)\left(y_{0}-x_{0}\right) d t .
$$


Using norm, we get

$$
\left\|z_{0}-x_{0}\right\| \leq\left(1+\frac{5 b_{0}}{1+\alpha}\right)\left\|y_{0}-x_{0}\right\|
$$

Similarly, $\left\|z_{0}-y_{0}\right\| \leq \frac{5 b_{0}}{1+\alpha}\left\|y_{0}-x_{0}\right\|$. Now

$$
x_{1}-x_{0}=-\Gamma_{0} F\left(x_{0}\right)-\frac{9}{5} \Gamma_{0} F\left(y_{0}\right)-\frac{1}{5} \Gamma_{0} F\left(z_{0}\right) .
$$

Using Taylor's expansion of $F\left(y_{0}\right)$ and $F\left(z_{0}\right)$ about $x_{0}$ and taking norm on both sides, we get

$$
\begin{aligned}
\left\|x_{1}-x_{0}\right\| & \leq\left\|\Gamma_{0} F\left(x_{0}\right)\right\|+\frac{4}{5}\left\|\Gamma_{0}\right\| \int_{0}^{1}\left\|F^{\prime}\left(x_{0}+t\left(y_{0}-x_{0}\right)\right)-F^{\prime}\left(x_{0}\right)\right\|\left\|y_{0}-x_{0}\right\| d t \\
& +\frac{1}{5}\left\|\Gamma_{0}\right\| \int_{0}^{1}\left\|F^{\prime}\left(x_{0}+t\left(z_{0}-x_{0}\right)\right)-F^{\prime}\left(x_{0}\right)\right\|\left\|z_{0}-x_{0}\right\| d t .
\end{aligned}
$$

Substituting (3.5) in (3.6), we get

$$
\begin{aligned}
\left\|x_{1}-x_{0}\right\| & \leq\left(1+\frac{4 b_{0}}{5(1+\alpha)}+\frac{b_{0}}{5(1+\alpha)}\left(1+\frac{5 b_{0}}{1+\alpha}\right)^{1+\alpha}\right)\left\|y_{0}-x_{0}\right\| \\
& \leq\left(1+\xi\left(b_{0}\right)\right)\left\|y_{0}-x_{0}\right\| .
\end{aligned}
$$

Assuming that $x_{n}, y_{n}, z_{n} \in D$ and $b_{0}<s_{0}$, then, recurrence relations given below can be easily shown by mathematical induction for $n \geq 1$.

(I) $\quad\left\|\Gamma_{n}\right\| \leq \psi\left(b_{n-1}\right)\left\|\Gamma_{n-1}\right\|$,

(II) $\quad\left\|y_{n}-x_{n}\right\| \leq\left\|\phi\left(b_{n-1}\right) \psi\left(b_{n-1}\right)\right\| y_{n-1}-x_{n-1} \|$,

(III) $\quad\left\|z_{n}-y_{n}\right\| \leq \frac{5}{1+\alpha} b_{n}\left\|y_{n-1}-x_{n-1}\right\|$,

(IV) $\quad\left\|\Gamma_{n}\right\| \omega\left(\left\|y_{n}-x_{n}\right\|\right) \leq b_{n}$,

(V) $\quad\left\|x_{n}-x_{n-1}\right\| \leq\left(1+\xi\left(b_{n-1}\right)\right)\left\|y_{n-1}-x_{n-1}\right\|$.

Consider

$$
\left\|I-\Gamma_{0} F^{\prime}\left(x_{1}\right)\right\| \leq\left\|\Gamma_{0}\right\|\left\|F^{\prime}\left(x_{1}\right)-F^{\prime}\left(x_{0}\right)\right\| \leq \beta \omega\left(\left\|x_{1}-x_{0}\right\|\right) .
$$

Using (3.7), we get $\left\|I-\Gamma_{0} F^{\prime}\left(x_{1}\right)\right\| \leq \beta\left(1+\xi\left(b_{0}\right)\right)^{\alpha} \omega(\eta) \leq b_{0}\left(1+\xi\left(b_{0}\right)\right)^{\alpha}<1$. From Banach Lemma, we get $\Gamma_{1}$ exists and

$$
\left\|\Gamma_{1}\right\| \leq \frac{1}{1-b_{0}\left(1+\xi\left(b_{0}\right)\right)^{\alpha}}\left\|\Gamma_{0}\right\|=\phi\left(b_{0}\right)\left\|\Gamma_{0}\right\| .
$$

Using Taylor's expansion of $F\left(x_{1}\right)$, we get

$$
F\left(x_{1}\right)=F\left(y_{0}\right)+F^{\prime}\left(y_{0}\right)\left(x_{1}-y_{0}\right)+\int_{y_{0}}^{x_{1}}\left(F^{\prime}(x)-F^{\prime}\left(y_{0}\right)\right) d x
$$

Now,

$$
\left\|F\left(y_{0}\right)\right\| \leq \frac{1}{1+\alpha} \omega(\eta)\left\|y_{0}-x_{0}\right\|
$$




$$
\begin{aligned}
\left\|F^{\prime}\left(y_{0}\right)\left(x_{1}-y_{0}\right)\right\| & \leq\left(b_{0}+1\right)\left\|\frac{9}{5} F\left(y_{0}\right)+\frac{1}{5} F\left(z_{0}\right)\right\| \\
& \leq \frac{\left(b_{0}+1\right)}{\beta} \xi\left(b_{0}\right)\left\|y_{0}-x_{0}\right\|,
\end{aligned}
$$

and

$$
\left\|\int_{y_{0}}^{x_{1}}\left(F^{\prime}(x)-F^{\prime}\left(y_{0}\right)\right) d x\right\| \leq \frac{1}{1+\alpha} \xi\left(b_{0}\right)^{1+\alpha} \omega\left(\left\|y_{0}-x_{0}\right\|\right)\left\|y_{0}-x_{0}\right\| .
$$

Taking norm on (3.9) and using (3.10),(3.11) and (3.12), we get

$$
\begin{aligned}
\left\|F\left(x_{1}\right)\right\| & \leq \frac{1}{\beta}\left(\frac{b_{0}}{1+\alpha}+\left(1+b_{0}\right) \xi\left(b_{0}\right)+\frac{b_{0} \xi\left(b_{0}\right)^{1+\alpha}}{1+\alpha}\right)\left\|y_{0}-x_{0}\right\| \\
& \leq \frac{1}{\beta} \psi\left(b_{0}\right)\left\|y_{0}-x_{0}\right\| .
\end{aligned}
$$

Using (3.8) and (3.13), we get

$$
\left\|y_{1}-x_{1}\right\| \leq\left\|\Gamma_{1}\right\|\left\|F\left(x_{1}\right)\right\| \leq \phi\left(b_{0}\right)\left\|\Gamma_{0}\right\|\left\|F\left(x_{1}\right)\right\| \leq \phi\left(b_{0}\right) \psi\left(b_{0}\right)\left\|y_{0}-x_{0}\right\| .
$$

Now,

$$
\begin{aligned}
\left\|z_{1}-y_{1}\right\| & \leq 5\left\|\Gamma_{1}\right\| F\left(y_{1}\right)\|\| \leq 5\left\|\Gamma_{1}\right\| \int_{0}^{1}\left\|F^{\prime}\left(x_{1}+t\left(y_{1}-x_{1}\right)\right)-F^{\prime}\left(x_{1}\right)\right\|\left\|y_{1}-x_{1}\right\| d t \\
& \leq 5 \phi\left(b_{0}\right)\left\|\Gamma_{0}\right\| \frac{1}{1+\alpha} \omega\left(\left\|y_{1}-x_{1}\right\|\right)\left\|y_{1}-x_{1}\right\| \\
& \leq \frac{5}{1+\alpha} \beta \phi\left(b_{0}\right) \phi\left(b_{0}\right)^{\alpha} \psi\left(b_{0}\right)^{\alpha} \omega\left(\left\|y_{0}-x_{0}\right\|\right)\left\|y_{1}-x_{1}\right\| \\
& \leq \frac{5}{1+\alpha} \beta \omega\left(\left\|y_{0}-x_{0}\right\|\right) \phi\left(b_{0}\right)^{1+\alpha} \psi\left(b_{0}\right)^{\alpha}\left\|y_{1}-x_{1}\right\| \\
& \leq \frac{5}{1+\alpha} b_{0} \phi\left(b_{0}\right)^{1+\alpha} \psi\left(b_{0}\right)^{\alpha}\left\|y_{1}-x_{1}\right\| .
\end{aligned}
$$

This gives,

$$
\left\|z_{1}-y_{1}\right\| \leq \frac{5}{1+\alpha} b_{1}\left\|y_{1}-x_{1}\right\|
$$

Using (3.8) and (3.14), we get

$$
\begin{aligned}
\left\|\Gamma_{1}\right\| \omega\left(\left\|y_{1}-x_{1}\right\|\right) & \left.\leq \phi\left(b_{0}\right)\left\|\Gamma_{0}\right\| \phi\left(b_{0}\right)\right)^{\alpha} \psi\left(b_{0}\right)^{\alpha} \omega\left(\left\|y_{0}-x_{0}\right\|\right) \\
& \left.\leq \beta \omega\left(\left\|y_{0}-x_{0}\right\|\right) \phi\left(b_{0}\right)\right)^{1+\alpha} \psi\left(b_{0}\right)^{\alpha} \\
& \leq b_{0} \phi\left(b_{0}\right)^{1+\alpha} \psi\left(b_{0}\right)^{\alpha}=b_{1} .
\end{aligned}
$$

This gives,

$$
\left\|\Gamma_{1}\right\| \omega\left(\left\|y_{1}-x_{1}\right\|\right) \leq b_{1} .
$$

Thus, the recurrence relations (I)-(IV) are proved for $n=1$ by (3.8), (3.14), (3.15) and (3.16) respectively. The recurrence relation $(\mathrm{V})$ is already proved in (3.7) for $n=1$. Suppose that $x_{k}, y_{k}, z_{k} \in D$, and (I)-(V) holds for $n=k$. In a similar manner, we can prove all the recurrence relations (I)-(IV) by mathematical induction for $n \geq 1$. 


\subsection{Convergence Theorem}

In this subsection, we shall give existence and uniqueness theorems for the convergence of the sequence generated by (1.5) to the solution $x^{*}$ of (1.1). The estimation of error bounds are also provided.

Theorem 3.1. Let $x_{0} \in D$ and the conditions (1), (2) and (3) hold. Let $b_{0}=\beta \omega(\eta)$ satisfies $b_{0}<s_{0}$ where, $s_{0}$ is the smallest positive root of $u(1+\xi(u))^{\alpha}-1, \alpha \in[0,1]$ and $\xi(u)$ is given by (3.4). Then, starting with $x_{0}$, the sequence $\left\{x_{n}\right\}$ generated by (1.5) converges to $x^{*}$ of (1.1) with $x_{n}, y_{n}, z_{n}, x^{*} \in \overline{B\left(x_{0}, R \eta\right)}$ where, $B\left(x_{0}, R \eta\right)=\left\{x \in X:\left\|x-x_{0}\right\|<R\right\} \subset D$, where $R=\frac{5}{1+\alpha} b_{0}+\frac{\left(1+\xi\left(b_{0}\right)\right)}{1-\phi\left(b_{0}\right) \psi\left(b_{0}\right)}$.

Proof. First of all, we have to prove that $y_{n}$ and $z_{n} \in \overline{B\left(x_{0}, R \eta\right)} \subset D$. Using recurrence relation (V), we get

$$
\begin{aligned}
\left\|x_{n}-x_{0}\right\| & \leq\left\|x_{n}-x_{n-1}\right\|+\ldots+\left\|x_{1}-x_{0}\right\| \\
& \leq\left(1+\xi\left(b_{n-1}\right)\right)\left\|y_{n-1}-x_{n-1}\right\|+\cdots+\left(1+\xi\left(b_{0}\right)\right)\left\|y_{0}-x_{0}\right\| \\
& \leq\left(1+\xi\left(b_{0}\right)\right) \sum_{k=0}^{n-1}\left(\phi\left(b_{0}\right) \psi\left(b_{0}\right)\right)^{k}\left\|y_{0}-x_{0}\right\| .
\end{aligned}
$$

Now, using recurrence relation (II) and (3.17), we get

$$
\begin{aligned}
\left\|y_{n}-x_{0}\right\| & \leq\left\|y_{n}-x_{n}\right\|+\left\|x_{n}-x_{0}\right\| \\
& \leq \phi\left(b_{n-1}\right) \psi\left(b_{n-1}\right)\left\|y_{n-1}-x_{n-1}\right\|+\left(1+\xi\left(b_{0}\right)\right) \sum_{k=0}^{n-1}\left(\phi\left(b_{0}\right) \psi\left(b_{0}\right)\right)^{k}\left\|y_{0}-x_{0}\right\| \\
& \leq\left(\phi\left(b_{0}\right) \psi\left(b_{0}\right)\right)^{n}\left\|y_{0}-x_{0}\right\|+\left(1+\xi\left(b_{0}\right)\right) \sum_{k=0}^{n-1}\left(\phi\left(b_{0}\right) \psi\left(b_{0}\right)\right)^{k}\left\|y_{0}-x_{0}\right\| \\
& \leq\left(1+\xi\left(b_{0}\right)\right) \sum_{k=0}^{n}\left(\phi\left(b_{0}\right) \psi\left(b_{0}\right)\right)^{k}\left\|y_{0}-x_{0}\right\| \\
& \leq\left(1+\xi\left(b_{0}\right)\right) \frac{1-\left(\phi\left(b_{0}\right) \psi\left(b_{0}\right)\right)^{n+1}}{1-\phi\left(b_{0}\right) \psi\left(b_{0}\right)}\left\|y_{0}-x_{0}\right\|<R \eta .
\end{aligned}
$$

Using recurrence relation (III) and (3.18), we get

$$
\begin{aligned}
\left\|z_{n}-x_{0}\right\| & \leq\left\|z_{n}-y_{n}\right\|+\left\|y_{n}-x_{0}\right\| \\
& \leq \frac{5}{1+\alpha} b_{0}\left(\phi\left(b_{0}\right) \psi\left(b_{0}\right)\right)^{n}\left\|y_{0}-x_{0}\right\|+\left(1+\xi\left(b_{0}\right)\right)^{\alpha} \frac{1-\left(\phi\left(b_{0}\right) \psi\left(b_{0}\right)\right)^{n+1}}{1-\phi\left(b_{0}\right) \psi\left(b_{0}\right)}\left\|y_{0}-x_{0}\right\| \\
& \leq\left(\frac{5}{1+\alpha} b_{0}+\left(1+\xi\left(b_{0}\right)\right)^{\alpha} \frac{1-\left(\phi\left(b_{0}\right) \psi\left(b_{0}\right)\right)^{n+1}}{1-\phi\left(b_{0}\right) \psi\left(b_{0}\right)}\right)\left\|y_{0}-x_{0}\right\|<R \eta .
\end{aligned}
$$


Now,

$$
\begin{aligned}
\left\|x_{n+1}-x_{n}\right\| & \leq\left(1+\xi\left(b_{n}\right)\right)\left\|y_{n}-x_{n}\right\| \\
\leq & \left(1+\xi\left(b_{n}\right)\right) \phi\left(b_{n-1}\right) \psi\left(b_{n-1}\right)\left\|y_{n-1}-x_{n-1}\right\| \\
& \leq \cdots \leq\left(1+\xi\left(b_{n}\right)\right)\left(\prod_{j=0}^{n-1} \phi\left(b_{j}\right) \psi\left(b_{j}\right)\right)\left\|y_{0}-x_{0}\right\| .
\end{aligned}
$$

Now, to ensure the convergence of $\left\{x_{n}\right\}$, we show that $\left\{x_{n}\right\}$ is a Cauchy sequence. Consider

$$
\begin{aligned}
\left\|x_{n+k}-x_{n}\right\| & \leq\left\|x_{n+k}-x_{n+k-1}\right\|+\ldots+\left\|x_{n+1}-x_{n}\right\| \\
& \leq\left(1+\xi\left(b_{n+k-1}\right)\right)\left(\prod_{j=0}^{n+k-2} \phi\left(b_{j}\right) \psi\left(b_{j}\right)\right) \eta+\ldots+\left(1+\xi\left(b_{n}\right)\right)\left(\prod_{j=0}^{n-1} \phi\left(b_{j}\right) \psi\left(b_{j}\right)\right) \eta \\
& \leq\left(1+\xi\left(b_{0}\right)\right) \sum_{l=0}^{k-1}\left(\prod_{j=0}^{n+l-1} \phi\left(b_{j}\right) \psi\left(b_{j}\right)\right) \eta \\
& \leq\left(1+\xi\left(b_{0}\right)\right) \sum_{l=0}^{k-1}\left(\phi\left(b_{0}\right) \psi\left(b_{0}\right)\right)^{l+n} \eta .
\end{aligned}
$$

This gives,

$$
\left\|x_{n+k}-x_{n}\right\| \leq\left(1+\xi\left(b_{0}\right)\right) \frac{1-\left(\phi\left(b_{0}\right) \psi\left(b_{0}\right)\right)^{k}}{1-\phi\left(b_{0}\right) \psi\left(b_{0}\right)}\left(\phi\left(b_{0}\right) \psi\left(b_{0}\right)\right)^{n} \eta .
$$

Hence $\left\{x_{n}\right\}$ is a Cauchy sequence since, $\phi\left(b_{0}\right) \psi\left(b_{0}\right)<1$ from Lemma 3.2 and hence, it is convergent. For $n=0$ and $k \geq 1$, we get

$$
\left\|x_{k}-x_{0}\right\| \leq\left(1+\xi\left(b_{0}\right)\right) \frac{1-\left(\phi\left(b_{0}\right) \psi\left(b_{0}\right)\right)^{k}}{1-\phi\left(b_{0}\right) \psi\left(b_{0}\right)}<R \eta .
$$

Thus, $x_{k} \in \overline{B\left(x_{0}, R \eta\right)}$. Taking $k \rightarrow \infty$ in (3.19), we obtain $x^{*} \in \overline{B\left(x_{0}, R \eta\right)}$. Now, it is to be shown that $x^{*}$ is a solution of (1.1). From (1.5), we have $y_{n}=x_{n}-\Gamma_{n} F\left(x_{n}\right)$. This gives

$$
\begin{aligned}
\left\|F\left(x_{n}\right)\right\| & \leq\left\|F^{\prime}\left(x_{n}\right)\right\|\left\|y_{n}-x_{n}\right\| \\
& \leq\left\|F^{\prime}\left(x_{n}\right)\right\| \phi\left(b_{n-1}\right) \psi\left(b_{n-1}\right)\left\|y_{n-1}-x_{n-1}\right\| \\
& \leq\left\|F^{\prime}\left(x_{n}\right)\right\|\left(\phi\left(b_{0}\right) \psi\left(b_{0}\right)\right)^{n}\left\|y_{0}-x_{0}\right\| .
\end{aligned}
$$

Now,

$$
\begin{aligned}
\left\|F^{\prime}\left(x_{n}\right)\right\| & \leq\left\|F^{\prime}\left(x_{n}\right)-F^{\prime}\left(x_{0}\right)\right\|+\left\|F^{\prime}\left(x_{0}\right)\right\| \\
& \leq \omega\left(\left\|x_{n}-x_{0}\right\|\right)+\left\|F^{\prime}\left(x_{0}\right)\right\| \\
& <R^{\alpha} \omega(\eta)+\left\|F^{\prime}\left(x_{0}\right)\right\| .
\end{aligned}
$$

Therefore, $\left\|F^{\prime}\left(x_{n}\right)\right\|$ is bounded. Hence from (3.20), we obtain that $\left\|F\left(x_{n}\right)\right\| \rightarrow 0$ as $n \rightarrow \infty$. Thus by using continuity of $F$ in $D$, we get $F\left(x^{*}\right)=0$. 
Next theorem gives the uniqueness of $x^{*}$.

Theorem 3.2. If $r$ be a positive root of

$$
\frac{2 \beta}{1+\alpha} \omega(R+r)\left(1-\frac{1}{2^{1+\alpha}}\right)=1
$$

then from theorem 3.1, the solution $x^{*}$ is unique in $B\left(x_{0}, r\right) \cap D$.

Proof. In order to prove the uniqueness part, suppose there exists $s^{*} \in B\left(x_{0}, r\right)$ such that $F\left(s^{*}\right)=0, s^{*} \neq x^{*}$. Then

$$
0=F\left(s^{*}\right)-F\left(x^{*}\right)=\int_{0}^{1} F^{\prime}\left(x^{*}+\theta\left(s^{*}-x^{*}\right)\right) d \theta\left(s^{*}-x^{*}\right)=T\left(s^{*}-x^{*}\right),
$$

where $T=\int_{0}^{1} F^{\prime}\left(x^{*}+\theta\left(s^{*}-x^{*}\right)\right) d \theta$. Consider

$$
\begin{aligned}
\left\|I-\Gamma_{0} T\right\| & \leq\left\|\Gamma_{0}\right\| \int_{0}^{1}\left\|\left(F^{\prime}\left(x^{*}+\theta\left(s^{*}-x^{*}\right)\right)-F^{\prime}\left(x_{0}\right)\right)\right\| d \theta \\
& \leq \beta \int_{0}^{1} \omega\left(\left\|x^{*}+\theta\left(s^{*}-x^{*}\right)-x_{0}\right\|\right) d \theta \\
& \leq \beta \int_{0}^{1} \omega\left(\left\|(1-\theta)\left(x^{*}-x_{0}\right)+\theta\left(s^{*}-x_{0}\right)\right\|\right) d \theta \\
& <\beta \int_{0}^{1 / 2}(1-\theta)^{\alpha} \omega(R+r) d \theta+\beta \int_{1 / 2}^{1} \theta^{\alpha} \omega(R+r) d t \\
& =2 \beta \omega(R+r) \int_{1 / 2}^{1} \theta^{\alpha} d t \\
& =\frac{2 \beta}{1+\alpha} \omega(R+r)\left(1-\frac{1}{2^{1+\alpha}}\right)=1 .
\end{aligned}
$$

Thus, $\left\|I-\Gamma_{0} T\right\|<1$. Therefore, $T^{-1}$ exists and hence $s^{*}=x^{*}$.

\subsection{Numerical examples}

In this subsection, examples are solved to demonstrate the applicability of our work.

Example 3.1. Consider the following nonlinear Hammerstein type integral equation

$$
x(r)=1+\int_{0}^{1} K(r, s)\left(x(s)^{7 / 5}+\frac{x(s)^{2}}{10}\right) d s,
$$

where, $K(r, s)$ denotes Green's function given by

$$
K(r, s)= \begin{cases}r(1-s), & r \leq s \\ s(1-r), & s \leq r .\end{cases}
$$


From (3.22), we get,

$$
F(x)(r)=x(r)-1-\int_{0}^{1} K(r, s)\left(x(s)^{7 / 5}+\frac{x(s)^{2}}{10}\right) d s,
$$

where $F: D \subseteq C[0,1] \rightarrow C[0,1]$. Therefore

$$
F^{\prime}(x) y(r)=y(r)-\int_{0}^{1} K(r, s)\left(\frac{7}{5} x(s)^{2 / 5}+\frac{x(s)}{5}\right) y(s) d s .
$$

This gives

$$
\left\|F^{\prime}(x)-F^{\prime}(y)\right\| \leq \frac{1}{40}\left(7\|x-y\|^{2 / 5}+\|x-y\|\right) .
$$

One can easily show the failure of Lipschitz and Hölder conditions and the success of $\omega$-condition. Here, $\omega(x)=\frac{1}{40}\left(7 x^{2 / 5}+x\right)$. For $x_{0}(r)=1$, we obtain $\beta=5 / 4, \eta=11 / 64$ and $\alpha=2 / 5$. Thus, the conditions of Lemma 1, Lemma 2 and Theorem 2.1 are satisfied. Hence the existence of $x^{*}$ is guaranteed in $\overline{B\left(x_{0}, 0.30701\right)}$ and the uniqueness in $B\left(x_{0}, 15.14198\right)$.

Example 3.2. Consider the following nonlinear Hammerstein type integral equation

$$
x(r)=1+\int_{0}^{1} K(r, s) x(s)^{3 / 2} d s,
$$

where, $K(r, s)$ denotes the Green's function in $[0,1]$. Therefore,

$$
F(x)(r)=x(r)-1-\int_{0}^{1} K(r, s) x(s)^{3 / 2} d s .
$$

Clearly,

$$
F^{\prime}(x) y(r)=y(r)-\frac{3}{2} \int_{0}^{1} K(r, s) x(s)^{1 / 2} y(s) d s
$$

This gives

$$
\left\|F^{\prime}(x)-F^{\prime}(y)\right\| \leq \frac{3}{16}\|x-y\|^{1 / 2}
$$

Thus, Lipschitz condition on $F^{\prime}$ fails but Hölder and $\omega$-conditions hold. Here, $\omega(x)=\frac{3}{16} x^{1 / 2}$. For $x_{0}(r)=1$, we obtain $\beta=\frac{16}{13}, \eta=\frac{2}{13}$ and $\alpha=\frac{1}{2}$. Therefore, the conditions of Lemma 3.1, Lemma 3.2 and Theorem 3.1 are satisfied. Hence $x^{*}$ exists in $\overline{B\left(x_{0}, 0.23879\right)}$ and unique in $B\left(x_{0}, 25.03679\right)$. To get numerical solution, we approximate the following integral by using thee Gauss-Legendre formula given by

$$
\int_{0}^{1} \zeta(t) d t \simeq \frac{1}{2} \sum_{j=1}^{m} \beta_{j} \zeta\left(t_{j}\right),
$$


where $\beta_{j}$ and $t_{j}$ denote the weights and nodes respectively and are to be determined. If $x_{i}$ stands for $x\left(t_{i}\right), i=1,2, \ldots n$, then (3.24) gives

$$
x_{i}=1+\sum_{j=1}^{n} a_{i j} x_{j}^{3 / 2}, \quad i=1,2, \ldots m
$$

where,

$$
a_{i j}= \begin{cases}\frac{1}{2} \beta_{j} t_{j}\left(t_{i}-1\right) & \text { if } j \leq i \\ \frac{1}{2} \beta_{j} t_{i}\left(t_{j}-1\right) & \text { if } j>i\end{cases}
$$

Thus, (3.26) gives

$$
x=1+B x^{3 / 2},
$$

where, $B=\left(b_{i j}\right), x=\left(x_{1}, x_{2}, \ldots, x_{n}\right)^{T}$ and $x^{3 / 2}=\left(x_{1}^{3 / 2}, x_{2}^{3 / 2}, \ldots, x_{n}^{3 / 2}\right)^{T}$. The Table 1 gives the value of weights and nodes for $m=8$.

Table 1: Value of weights and nodes for $\mathrm{m}=8$

\begin{tabular}{|c|c|c|c|c|c|c|c|c|}
\hline$j$ & 1 & 2 & 3 & 4 & 5 & 6 & 7 & 8 \\
\hline$\beta_{j}$ & 0.1012 & 0.2238 & 0.3137 & 0.3627 & 0.3627 & 0.3137 & 0.2238 & 0.1012 \\
$t_{j}$ & 0.0198 & 0.1016 & 0.2372 & 0.4083 & 0.5917 & 0.7627 & 0.8983 & 0.9801 \\
\hline
\end{tabular}

Table 2: Solution of (3.26)

\begin{tabular}{|c|c|c|c|c|c|c|c|c|}
\hline$j$ & 1 & 2 & 3 & 4 & 5 & 6 & 7 & 8 \\
\hline$x_{j}$ & 0.9911 & 0.9587 & 0.9192 & 0.8930 & 0.8930 & 0.9192 & 0.9587 & 0.9911. \\
\hline
\end{tabular}

Starting with $x_{0}$, we can approximate the solution by (1.5) in the following manner.

$$
\begin{aligned}
F^{\prime}\left(x_{n}\right) a_{n} & =-F\left(x_{n}\right) \\
y_{n} & =x_{n}+a_{n} \\
F^{\prime}\left(x_{n}\right) c_{n} & =-F\left(y_{n}\right) \\
z_{n} & =y_{n}+5 c_{n} \\
F^{\prime}\left(x_{n}\right) d_{n} & =-F\left(z_{n}\right) \\
x_{n+1} & =z_{n}-\frac{16}{5} c_{n}+\frac{1}{5} d_{n} .
\end{aligned}
$$

Figure 1: Approximate solution of (3.26)

The numerical solution is displayed in the Table 2 for $x_{0}=(1,1, \ldots, 1)^{T}$ and $m=8$. In the Figure 1, interpolating function passing through $\left(t_{i}, x_{i}\right)$, is an approximate solution of (3.26). 


\section{Conclusions}

Local and semilocal convergence of an iteration of fifth order is established under weaker Argyros-type and $\omega$-conditions on $F^{\prime}$ for finding the solutions of nonlinear equations in Banach spaces. This avoids derivatives of higher order which are either expensive to compute or are unbounded. Existence and uniqueness theorems and the error bounds for the solution are provided. A number of examples including Hammerstein type nonlinear integral equation are tested to demonstrate the applicability of our approach.

\section{References}

[1] A. Cordero, J.A. Ezquerro, M.A. Hernández-Verón, J.R. Torregrosa, On the local convergence of a fifth-order iterative method in Banach spaces, Appl. Math. Comput. 251 (2015) 396-403.

[2] A. Cordero, M.A. Hernández-Verón, N. Romero and J.R. Torregrosa; Semilocal convergence by using recurrence relations for a fifth-order method in Banach spaces, J. Comput. Appl. Math. 273 (2015) 205-213.

[3] S. Singh, D.K. Gupta, E. Martínez, J.L. Hueso, Semilocal and local convergence of a fifth order iteration with Fréchet derivative satisfying Hölder condition, Appl. Math. Comput. 276 (2016) 266-277.

[4] M.A. Hernández, E. Martínez, On the semilocal convergence of a three steps Newton-type iterative process under mild convergence conditions, Numer. Algorithms 70 (2015) 377-392.

[5] M. Prashant, D. K. Gupta, A continuation method and its convergence for solving nonlinear equations in Banach spaces, International journal of Computational Methods 10 (2013) 123.

[6] S.K. Parhi, D. K. Gupta, Semilocal Convergence of Stirling-Like Method in Banach spaces, International journal of Computational Methods 7 (2010) 215-228.

[7] X. Wang, J. Kou, Semilocal convergence of multi-point improved super-Halley-type methods without second derivative under generalized weak condition, Numer. Algorithms (2015), DOI 10.1007/s11075-015-0010-x.

[8] V. Arroyo, A. Cordero and J.R. Torregrosa; Approximation of artifical satellites' preliminary orbits: The efficiency challenge, Math. Comput. Model., Vol-54 (2011) pp. 1802-1807.

[9] I.K. Argyros, S. Hilout, Numerical methods in Nonlinear Analysis, World Scientific Publ. Comp. New Jersey 2013.

[10] I.K. Argyros, S. Hilout, M.A. Tabatabai, Mathematical Modelling with Applications in Biosciences and Engineering, Nova Publishers, New York, 2011. 
[11] M. Ganesh, M.C. Joshi, Numerical solvability of Hammerstein integral equations of mixed type, IMA. J. Numer. Anal. 11 (1991) 21-31.

[12] J.L. Hueso, E. Martínez, Semilocal convergence of a family of iterative methods in Banach spaces, Numer. Algorithms 67 (2014) 365-384.

[13] J.A. Ezquerro, M.A. Hernández, A modification of the super-Halley method under mild differentiabilty conditions, J. Comput. Appl. Math. 114 (2000) 405-409.

[14] J.A. Ezquerro, M.A. Hernández, On Halley-type iterations with free second derivative, J. Comput. Appl. Math. 170 (2004) 455-459.

[15] J.A. Ezquerro, M.A. Hernández, On the R-order of the Halley method, J. Math. Anal. Appl. 303 (2005) 591-601.

[16] X. Ye, C. Li, W. Shen, Convergence of the varints of the Chebyshev-Halley iteration family under Hölder condition of the first derivative, J. Comput. Appl. Math. 203 (2007) 279-288.

[17] I.K. Argyros, J. Chen, On the local convergence of a Newton-type method in Banach spaces, Int. J. Comput. Math. 86 (2009) 1366-1374.

[18] I.K Argyros, S.K.Khattri, Local convergence for a family of third order methods in Banach spaces, Journal of Mathematics, 46 (2014) 53-62.

[19] C. Chun, P. Stănică, B. Neta, Third-order family of methods in Banach spaces, Comput. Math. Appl. 61 (2011) 1665-1675.

[20] I.K. Argyros, P. Jidesh, S. George, Ball Convergence for Second Derivative Free Methods in Banach Space, Int. J. Appl. Comput. Math (2015) DOI 10.1007/s40819-015-0125-8.

[21] I.K. Argyros, A. S. Hilout, On the local convergence of fast two-step Newton-like methods for solving nonlinear equations, J. Comput. Appl. Math. 245 (2013) 1-9.

[22] M.A. Hernández, The Newton method for operators with Holder continuous first derivative, J. Optim. Theory Appl. 109 (2001) 631-648.

[23] I.K. Argyros, S. George, Local convergence for deformed Chebyshev-type method in Banach space under weak conditions, Cogent Mathematics 2 (2015) 1-12.

[24] I.K. Argyros, S. George, Local convergence of deformed Halley method in Banach space under Holder continuity conditions, J. Nonlinear Sci. Appl. 8 (2015) 246-254.

[25] S.K. Parhi, D.K. Gupta, Relaxing convergence conditions for Stirling's method, Math. Methods Appl. Sci. 32 (2010) 224-232. 
[26] S.K. Parhi, D.K. Gupta, Convergence of a third order method for fixed points in Banach spaces, Numer. Algorithms 60 (2012) 419-434.

[27] L.V. Kantorovich, G.P. Akilov, Functional Analysis, Pergamon Press, Oxford,1982.

[28] I.K. Argyros, S. George, A. A. Magreñán, Local convergence for multi-point-parametric Chebyshev-Halley-type methods of higher convergence order, J. Comput. Appl. Math. 282 (2015) 215-224.

[29] I.K. Argyros and A. A. Magreñán, A study on the local convergence and the dynamics of Chebyshev-Halley-type methods free from second derivative, Numer. Algorithms DOI 10.1007/s11075-015-9981-x.

[30] S. Amat, M.A. Hernández and N. Romero; A modified Chebyshev's iterative method with at least sixth order of convergence, Appl. Math. Comput. 206 (2008) 164-174.

[31] J.A. Ezquerro, M.A. Hernández, M.A. Salanova, A Newton-like method for solving some boundary value problems, Numer. Func. Anal. Opt. 23 (2002) 791-805.

[32] P.K. Parida, D.K. Gupta, Recurrence relations for semilocal convergence of a Newton-like method in Banach spaces, J. Math. Anal. Appl. 345 (2008) 350-361.

[33] A. Kumar, D.K. Gupta, Local convergence of Super Halley's method under weaker conditions on Frechet derivative in Banach spaces, J. Anal. DOI 10.1007/s41478-017-0034-9 